geschehen mit grundsätzlicher Abhängigkeit vom Ploidie-Grad ist, nur daß eben durch Überlagerungserscheinungen, wie sie bei UV-Strahlen als Noxe auftreten, das dem jeweiligen Ploidie-Grad entsprechende Treffergeschehen zur Unkenntlichkeit verzerrt wird. So kann also für UV-Strahlen angenommen werden, daß sie auf den Vererbungsmechanismus zwar im Grunde genommen dem Prinzip nach wie andere Noxen einwirken, daß jedoch bei ihnen eine Analyse nach dem Treffergesetz nicht in allen Fällen durchführbar ist, weil die dem Absterben zugrunde liegende Primärerscheinung, die den „normalen" Reaktionsablauf bestimmt, hier u. U. durch Überlagerungen von anderen Reaktionsabläufen verdeckt wird.

Diese UV-Befunde bedeuten also keine Durchbrechung der erstgemachten Feststellung, daß prinzipiell auch für UV-Strahlen die gleichen Gesetzmäßigkeiten, wie sie für die anderen Noxen ermittelt wurden, gelten. Wie die hier gemachten Untersuchungen und Überlegungen zeigen, sind aber die UV-Strahlen als Noxe nicht in allen Fällen für eine Analyse nach dem Treffergesetz geeignet. Es liegt selbstverständlich durchaus im Bereich des Möglichen, daß es auch noch andere Noxen geben wird, die ebenfalls auf Grund von Überlagerungserscheinungen für derartige Analysen nicht verwendbar sind.

$\mathrm{Da}$ also die Charakteristik der Absterbekurven, wie in den untersuchten Fällen gezeigt werden konnte, bei Einzellern unabhängig von der Noxe (unter Ausschluß von UV) prinzipiell vom PloidieGrad abhängen dürfte, müßte es mit Hilfe von Absterbekurven auch möglich sein, Aussagen über den Ploidie-Grad bei Einzellern zu machen. Hiervon machte $\mathrm{Magni}{ }^{23}$ bereits bei Untersuchungen mit Röntgenstrahlen Gebrauch. Bei unseren Untersuchungen konnte mit chemischen Noxen für einige Torula-Arten auf Grund der Absterbekurven (Eintreffer-Kurven) bestätigt werden, daß es sich bei den untersuchten Fällen um haploide Organismen handelt, wofür auch die Kopulationsversuche von Wickerham und Burton ${ }^{11}$ sprechen. Demgegenüber reagierte Candida utilis, ein Organismus, der erst in neuerer Zeit von Torulopsis abgetrennt wurde, nach einem Zwei-Eintreffer-Geschehen. Diese Candida-Art müßte demnach diploid sein, ein weiterer Umstand, der ihre Abtrennung von Torulopsis rechtfertigt.

In vielen Fällen, in denen bisher vererbungs- und zytogenetische Untersuchungsmethoden zur Bestimmung des Ploidie-Grades bei Einzellern zu keinem sicheren Ergebnis führten, dürfte es daher auf Grund einer Analyse nach dem Treffergesetz nunmehr möglich sein, Aussagen über den Ploidie-Grad zu machen.

${ }^{23}$ G. E. M a g n i, Atti Ist. bot. ecc. Pavia, Ser. V, 10, 89 [1953].

\title{
Beziehungen zwischen Wachstum und Formbildung in Abhängigkeit von Licht und Temperatur bei Acetabularia*
}

\section{Von Kurt Beth}

Aus dem Max-Planck-Institut für Meeresbılogie, Abt. H ä m m e rling, Wilhelmshaven (Z. Naturforschg. 10 b, 267-276 [1955]; eingegangen am 3. Januar 1955)

Bei mehreren Acetabularia-Arten werden formative Licht- und Temperaturwirkungen auf das Hauptentwicklungsstadium der Einzelzelle, das von Kernteilungs-Vorgängen nicht beeinflußt ist, näher untersucht. Die Zellen zeigen nach Aufzucht in verschiedenen Bedingungen bei der Hutanlage charakteristische Unterschiede in den Stiellängen. So sind die Stiele desto kürzer, je mehr Licht täglich geboten wird. Die endgültige Stiellänge erscheint als Resultante zweier Vorgänge, des Stielwachstums und der gleichzeitig ablaufenden, vorbereitenden Hutbildungsprozesse, deren Geschwindigkeit in verschiedenem Maße von Außenfaktoren beeinflußt werden. Die vorbereitenden Hutbildungsprozesse haben ein höheres Licht- und Temperaturbedürfnis als das Stielwachstum, das Stielwachstum stellt sich als eine „niedere“, das Hutwachstum als eine „höhere“ Form des Wachstums dar. Die Unterschiede betreffen Prozesse des Cytoplasmas. Die betreffenden Vorgänge können als wesentlich von cytoplasmatischen Prozessen bestimmt cngesehen werden.

F frühere Versuche zur Formbildung von Acetabularia ergaben, daß während des Stielwachstums vorbereitende Hutbildungs-Prozesse stattfinden ${ }^{1}$. Während die

* Mit Unterstützung der Deutschen Forschungsgemeinschaft für die in dieser Arbeit enthaltenen Probleme seit 1. 4.1953.
Stielwachstums-Vorgänge bei niedrigen Lichtintensitäten vollständig ablaufen können, verlangt eine (kern-unabhängige) Phase der vorbereitenden Hutbildungs-Prozesse 1 J. H ämmerling, Roux' Arch. Entwicklungsmechan. Organismen 131, 1 [1934]; Naturwissenschaften $33,337,361$ [1946]. 


\begin{tabular}{|c|c|c|r|}
\hline $\begin{array}{c}\text { Licht } \\
\text { Temp. }\end{array}$ & $\begin{array}{c}\text { Tages- } \\
\text { länge } \\
\text { [h] }\end{array}$ & $\begin{array}{c}\text { Stiellänge } \\
\text { [mm] }\end{array}$ & $(n)$ \\
\hline \begin{tabular}{c|c|c} 
Normal- \\
licht
\end{tabular} & 24 & 10,8 & $(56)$ \\
$21^{0}$ & 12 & 15,7 & $(121)$ \\
& 8 & 23,6 & $(51)$ \\
Normal- & 12 & 20,7 & $(155)$ \\
licht & 8 & 26,6 & $(53)$ \\
$27^{0}$ & 4 & 38,2 & $(26)$ \\
& 12 & 26,8 & $(62)$ \\
Schwach- & 8 & 31,3 & $(17)$ \\
licht & & & \\
$21^{0}$ & & & \\
\hline
\end{tabular}

Tab. 1. Acicularia schenckii. Stiellänge cystenhaltiger Pflanzen mit typischem Hut.

höhere Energiezufuhr 2. Im Folgenden sollen Experimente mitgeteilt werden, welche diesen Befund in einen größeren Zusammenhang einordnen. Es wurde untersucht, inwieweit abgestufte Licht- und Temperaturänderungen einerseits das Stielwachstum, andererseits die Hutbildung beeinflussen und dadurch auch die Größenverhältnisse der Zelle ändern.

\section{Material und Methode}

Die Versuche wurden entweder mit unzerschnittenen oder regenerierenden Zellen angestellt. Die ersteren kamen als junge Keimlinge oder junge, weniger als $1 \mathrm{~cm}$ lange Pflänzchen in die verschiedenen Versuchsbedingungen und wurden dort aufgezogen. Die regenerierenden Zellen stammten von Hutpflanzen aus normalen Kulturbedingungen; bei ihnen wurden zu Versuchsbeginn Stiel und Hut abgeschnitten, so daß nur das kernhaltige Rhizoid übrig blieb.

Die in Nordseewasser mit Zusätzen von Phosphat, Nitrat und Erdabkochung kultivierten Versuchspflanzen standen an künstlichen Sonnen bei $27^{\circ}\left( \pm 1^{\circ}\right)$ oder bei $21-22^{\circ}$ (nur kurzfristig $19^{\circ}$, ausnahmsweise bis $26^{\circ}$ ). Verschiedene Lichtintensitäten wurden erzielt durch Beleuchtung mit Leuchtstoffröhren (Normallicht etwa 2500 Lux) oder Glühlampen zu 60, 300 und 1000 Watt (Schwachlicht etwa 600 Lux, Normallicht alter Art etwa 2500 Lux, Starklicht etwa 5000 Lux).

Das Versuchsmaterial stammt aus den hiesigen Acetabularia-Kulturen. Besonders erwähnt werden muß Acicularia schenckii. Es wurden $\mathrm{f}_{1}$-Nachkommen von Pflanzen verwendet, die 1950 von $\mathrm{H} \ddot{\mathrm{a}} \mathrm{m}$ m e rling auf Bermuda gesammelt wurden. Sie kommen bei den normalen Kulturbedingungen $\left(21^{\circ}, 2500 \mathrm{Lux}\right)$ zur Hut- und Cystenbildung, wenn auch nicht ganz so gut wie die übrigen Acetabularia-Arten. Störungen treten besonders bei niedrigen Beleuchtungsmengen auf. Gameten entstehen in den Cysten jedoch bei $21^{\circ}$ nur spärlich, reichlich und prompt aber bei $27^{\circ}$. Die Bermuda-Pflanzen verhalten sich also anders als die früher in Curaçao gesammelten,

2 K. B eth, Z. Naturforschg. 8b, 334 [1953].

\begin{tabular}{|c|c|c|c|c|}
\hline & $\begin{array}{c}\text { Tempe- } \\
\text { ratur } \\
{\left[{ }^{0} \mathrm{C}\right]}\end{array}$ & $\begin{array}{c}\text { Tägliche } \\
\text { Beleuchtungs- } \\
\text { dauer } \\
{[\mathrm{h}]}\end{array}$ & Stiellänge \\
{$[\mathrm{mm}]$} & $n$ \\
\hline Regenerie- & 21 & 24 & 29,3 & 24 \\
rende Zellen & 21 & 12 & 28,9 & 24 \\
21 & 8 & 47,6 & 20 \\
& 27 & 12 & 23,8 & 22 \\
& 27 & 8 & 31,9 & 10 \\
Unbehandelte & 21 & 12 & 34,1 & 21 \\
Zellen & 21 & 6 & 43,0 & 20 \\
\hline
\end{tabular}

Tab. 2. A. mediterranea. Stiellänge unbehandelter und regenerierender Zellen, etwa 2500 Lux.

die bei Normalbedingungen überhaupt nicht zur Hutbildung kamen und sich wahrscheinlich durch ein höheres Lichtbedürfnis auszeichneten.

\section{Versuchsergebnis se}

\section{I.}

Werden Acetabularia-Pflanzen bei verschiedenen Licht- und Temperaturbedingungen aufgezogen, so zeigen die ausgewachsenen hut-haltigen Zellen kleine, aber regelmäßige Unterschiede in den Stiellängen.

Zum leichteren Verständnis des Folgenden sei kurz daran erinnert, daß die erwachsene Acetabularia-Zelle aus einem Stiel besteht, der einen oder mehrere Hüte (Gametangienstände) trägt und basal ein kleines Rhizoidknäuel aufweist, in dem der einzige Zellkern liegt. Das Stielwachstum wird mit der Anlage des Hutes vom Hutwachstum abgelöst. Es kann wieder einsetzen, indem der Hut durchwachsen wird. Dies erfolgt regelmäßig bei Arten, die, wie z. B. Acetabularia crenulata, mehrere Hüte bilden, ausnahmsweise aber auch bei Arten mit nur einem Hut. Unter endgültiger Stiellänge wird hier stets die Länge des Stieles zwischen Rhizoid und erstem Hut verstanden. Der Stiel verlängert sich nach der Hutbildung nur unwesentlich, bei A. wettsteinii und Polyphysa kann er schrumpfen 3 (vgl. Tab. 4). Das gesamte Zellwachstum einschließlich des Hutes ist spätestens mit der Teilung des Zellkernes und der darauffolgenden Cystenbildung beendet.

Bei allen untersuchten Arten wurde folgende Beziehung zwischen täglicher Lichtmenge und endgültiger Stiellänge gefunden: Erhöhung der Lichtmenge bewirkt Hutbildung bei kürzerer Stiellänge (Abb.1, Tab. 1-5). Dies gilt sowohl, wenn die Lichtmenge allein durch Verlängerung der täglichen Beleuchtungsdauer bei gleichbleibender Intensität erhöht wird, als auch wenn sie durch Intensitätssteigerung

3 J. Hämm e rling, Biol. Zbl. 54, 650 [1934]; Arch. Protistenkunde 97, 7 [1944]. 


\begin{tabular}{|c|c|c|}
\hline $\begin{array}{c}\text { Dauer der tägl. Beleuchtung } \\
{[\mathrm{h}]}\end{array}$ & $\begin{array}{c}\text { Stiellänge } \\
{[\mathrm{mm}]}\end{array}$ & $n$ \\
\hline 12 & 22,3 & 22 \\
6 & 28,8 & 21 \\
\hline
\end{tabular}

Tab. 3. A. crenulata. Stiellänge unbehandelter Zellen, etwa $2500 \mathrm{Lux}, 21^{\circ}$.

\begin{tabular}{|c|c|c|c|}
\hline $\begin{array}{c}\text { Dauer der } \\
\text { täglichen } \\
\text { Beleuchtung }\end{array}$ & $\begin{array}{c}|c| \\
\text { Stiellänge } \\
\text { bei Hutanlage } \\
\text { A } \\
{[\mathrm{h}]}\end{array}$ & $\begin{array}{c}\text { bei Cystenbildg. } \\
\mathrm{B}^{*} \\
{[\mathrm{~mm}]}\end{array}$ & $n$ \\
\hline & & & \\
12 & 1,5 & 0,6 & 6 \\
8 & 1,5 & 0,6 & 5 \\
4 & $3,0^{* *}$ & $1,5^{* *}$ & 5 \\
2 & $5,1^{* * *}$ & & 5 \\
\hline
\end{tabular}

* Stielverkürzung gegenüber $\mathrm{A}=$ Schrumpfungseffekt *** Vgl. auch Abb. 2 a

Tab. 4. A. wettsteinii. Stiellänge regenerierender Zellen bei Hutanlage und Cystenbildung (etwa 5000 Lux, 21ํ).
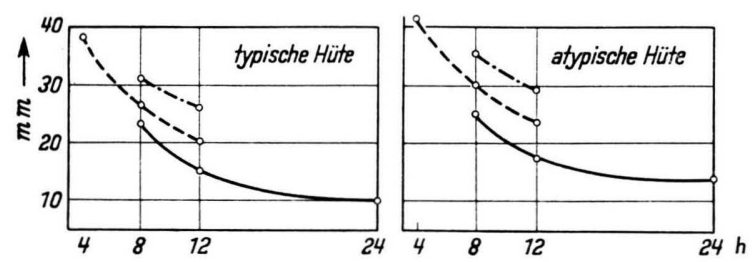

Abb.1. Acicularia schenckii. Stiellänge cystenhaltiger Hutpflanzen in Abhängigkeit von Temperatur und täglich gebotener Lichtmenge (Tageslänge und Intensität). a: Pflanzen mit typischem Hut, b: Pflanzen mit atypischem Hut. $-21^{\circ}$ Normallicht, $---27^{\circ}$ Normallicht, -.-.-.- $21^{\circ}$ Schwachlicht.

bei gleicher Tageslänge vermehrt wird, und gilt naturgemäß auch bei Kombination beider Faktoren. Diese Regel findet sich bestätigt bei Pflanzen mit typischen wie bei solchen mit atypischen Hüten (Tab. 1), ebenso bei unbehandelten und regenerierenden Pflanzen (Tab. 2)*

Während die Erhöhung der täglichen Lichtmenge bei allen untersuchten Arten einen gleichsinnigen Effekt hervorruft, ergeben die Temperatur-Versuche nicht dasselbe einheitliche Bild. Bei Acicularia und

* Bei gleichen Außenbedingungen unterscheiden sich unbehandelte und regenerierende Zellen in ihrer endgültigen Stiellänge (vgl. Tab. 2, $12 \mathrm{~h} 21^{\circ}$ ). Diesbezügliche Befunde brauchen in dieser Arbeit nicht berücksichtigt zu werden, sondern sollen in anderem Zusammenhang näher dargestellt werden.

\begin{tabular}{|c|c|c|c|}
\hline \multirow{2}{*}{$\begin{array}{c}\text { Dauer der } \\
\text { täglichen } \\
\text { Beleuchtung }\end{array}$} & \multicolumn{2}{|c|}{ Stiellänge } & \\
\cline { 2 - 3 }$[\mathrm{h}]$ & Extremwerte & Mittelwerte & $n$ \\
\hline & {$[\mathrm{mm}]$} & {$[\mathrm{mm}]$} & \\
\hline 24 & $1,2-4,3$ & $2,5^{*}$ & 165 \\
20 & $1,1-4,4$ & $2,4^{*}$ & 91 \\
16 & $1,6-6,6$ & $4,0^{*}$ & 86 \\
16 & $1,6-8,4$ & 4,2 & 208 \\
13 & $2,5-6,7$ & $4,5^{*}$ & 65 \\
13 & $2,9-8,5$ & 5,0 & 249 \\
8 & $9,2-16,0$ & 8,4 & 149 \\
\hline
\end{tabular}

Tab. 5. A. wettsteinii. Stiellänge huthaltiger Pflanzen (Schwachlicht, etwa $21^{\circ}$ ).

Bei den mit * bezeichneten Werten wurden nur die Stiellängen von cystenhaltigen Hutpflanzen ausgewertet, bei den übrigen auch Hutpflanzen ohne Cysten, mit noch nicht oder unvollständig geschrumpftem Stiel.
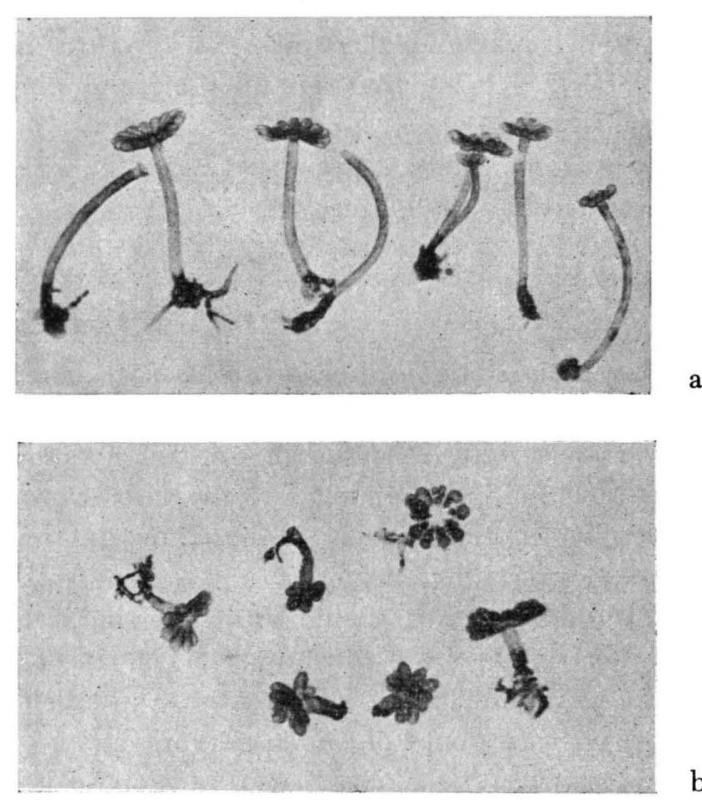

$\mathrm{b}$

Abb. 2. Acetabularia wettsteinii. Hutpflanzen bei $21^{\circ}$ und Starklicht. a: tägl. Beleuchtungsdauer $2 \mathrm{~h}$, b: tägl. Beleuchtungsdauer 4 Stunden.

A. crenulata hat Temperaturerhöhung um 6 auf $27^{\circ}$ Hutbildung bei längeren Stielen zur Folge, im Gegensatz zu mediterranea, welche bei $27^{\circ}$ kürzere Stiele als bei $21^{\circ}$ aufweist.

Die endgültigen Stiellängen zeigen bei den einzelnen Arten Unterschiede, die im übrigen durch geeignete Licht- und Temperaturbedingungen sicherlich noch vergrößert werden können. Beispielsweise 
liegen die Mittelwerte von Acicularia (Tab. 1) zwischen $11 \mathrm{~mm}\left(24 \mathrm{~h}, 2500 \mathrm{Lux}, 21^{\circ}\right)$ und $38 \mathrm{~mm}(4 \mathrm{~h}$, $2500 \mathrm{Lux}, 27^{\circ}$ ), die entsprechenden Einzelwerte zwischen 7 und $58 \mathrm{~mm}$. Die größten Unterschiede wurden bei A. wettsteinii gefunden. Einerseits kann man durch Starklicht (Tab. 4) Zellen erhalten, deren Stiel kleiner als $1 \mathrm{~mm}$ ist, deren Hut also unmittelbar auf dem Rhizoid zu sitzen scheint, andererseits können bei wenig Schwachlicht (Tab. 5) die Stiele über $1 \mathrm{~cm}$ lang werden (vgl. auch Abb. 2). Von ähnlicher Größenordnung sind die Unterschiede bei den übrigen untersuchten Arten.

Es sei darauf hingewiesen, daß die Veränderung der endgültigen Stiellänge auch eine gleichsinnige Veränderung der gesamten Zellgröße darstellt und nicht wesentlich durch gegensinnige Abweichung der Dimensionen anderer Zellteile (Hut- und Rhizoidgröße, Stieldurchmesser) kompensiert wird. Hierauf wird in der folgenden Arbeit näher eingegangen werden. Ferner sei erwähnt, daß die Stiellängen-Regeln auch bei Unregelmäßigkeiten im Stielzuwachs gelten (vgl. S. 272). Hinzugefügt sei außerdem, daß die Zellen selbst bei geringen Beleuchtungsmengen ein normales Aussehen hatten, zwar verlängert, aber offenbar nicht chlorophyll-ärmer waren.

II.

Der Zeitpunkt der Hutbildung bestimmt das Ende des bis dahir stetigen Stielwachstums und damit die endgültige Stiellänge. Diese ist also das Ergebnis zweier gleichzeitig ablaufender Vorgänge: des Stielwachstums und der einleitend erwähnten vorbereitenden Hutbildungs-Prozesse, die schon auf frühen Entwicklungsstadien beginnen und deren Abschluß zur Hutbildung führt. Beide Vorgänge sind, wie zu erwarten, in ihrer Geschwindigkeit von Licht und Temperatur abhängig, jedoch nicht in gleichem Maße, wie aus dem Folgenden hervorgeht.

* Sie darf nicht ohne weiteres als Maß der Substanzvermehrung hingenommen werden. Die auf Grund der m. o.w. gleichmäßigen Zunahme von Stiellänge und -durchmesser wahrscheinliche Vermutung, daß die Substanzvermehrung pro Zeiteinheit im Laufe der Entwicklung zunimmt, stimmt überein mit Messungen des Trokkengewichtes und Eiweißstickstoffes an kernhaltigen Teilen (W e rz, unveröffentl.). Allerdings zeigten die Bestimmungen $\mathrm{V}$ anderha e g h e s parallel zur Längenzunahme eine lineare Zunahme des Frischgewichtes und des Eiweißstickstoffes. Weitere Untersuchungen müssen die Verhältnisse genauer klären. Im übrigen berühren die Art und das Ausmaß der am Wachstumskomplex beteiligten Phänomene (z. B. Proteinsynthese, Zellstreckung durch Wasseraufnahme) die in dieser Arbeit angestellten Betrachtungen erst sekundär und bedürfen spezieller Untersuchungen.
1. a) Zuerst seien Versuche über das Stielwachstum in Abhängigkeit vom Licht dargestellt (vgl. Abb. 3 bis 10, Tab. 6 und 7). Als Wachstumsmaß wurde die Stielverlängerung genommen *. Allgemein ist $\mathrm{zu}$ sagen, daß durch Verlängerung der täglichen Beleuchtungsdauer, durch Intensitätssteigerung* sowie Temperaturerhöhung die Zellen schneller wachsen. Der Wachstumsverlauf ist bei einer bestimmten Versuchsbedingung m. o. w. geradlinig, d. h. der Stiel nimmt gleichmäßig an Länge und Durchmesser zu.

Abweichungen vom geradlinigen Kurvenverlauf kamen u. a. zu Versuchsbeginn vor und können wenigstens teilweise durch verzögerten Regenerationsbeginn und durch Unterschiede in den Anzucht- und Versuchsbedingungen erklärt werden. Bei einigen Kurven (Abb. 3, 6, 9, 10) fällt etwa im mittleren Kurventeil ein Knick und Steilerwerden auf. Diese Wachstumsbeschleunigung hängt jedoch mit einer zufälligen Temperaturerhöhung während der Versuche zusammen. Auf veränderte Außenbedingungen dürfen auch die Wachstumsverzögerungen bei den Kurven der Abb. 4 und 5 zurückgeführt werden.

$\mathrm{Ob}$ die Zunahme der Wachstumsgeschwindigkeit proportional der Tagesverlängerung ist, kann man der Neigung der verschiedenen Kurven nicht ohne weiteres ansehen, sondern muß berechnet werden. Bei allen Wachstumskurven wurde der tatsächliche Längenzuwachs in 10 Tagen an einem oder (bei Kurven mit verschiedenem Steilheitsgrad) an 2 Bereichen abgelesen und auf die gleiche Lichtstundenzahl von 120 (= 10 Tage je 12 Lichtstunden) umgerechnet. Die so erhaltenen Werte sind in Tab. 6 und 7 dargestellt. Zuerst sei A. crenulata (Tab. 6) besprochen. Die regenerierenden Zellen bieten klare Verhältnisse. Der Zuwachs ist im $12 \mathrm{~h}$ - Tag relativ am geringsten und nimmt allmählich über den $8 \mathrm{~h}-, 4 \mathrm{~h}-, 2 \mathrm{~h}$ - bis zum $1 \mathrm{~h}$ - Tag zu. Aus dem Versuch der Tab. 8, Sp. 2 und 3, geht hervor, daß die Pflanzen im 24h-Tag nicht schneller wachsen als im $12 \mathrm{~h}$ - Tag, der Zuwachs also nur halb so groß ist. Diese Tatsache fügt sich also gut ein. Der Vergleich von Normal- mit Starklicht ergibt, daß das schwächere Licht besser verwertet wird (Tab. 6, Sp. 2 und 3). Die dritte Versuchsserie

4 F. V a n d e rh a e ghe, Biochim. biophysica Acta [Amsterdam] 15, 281 [1954].

* Das Intensitätsoptimum wurde nicht bestimmt. $\mathrm{D}$ a $0^{5}$ fand für Acetabularia mediterranea ein Optimum von etwa 1300 Lux bei 11 Stdn. Beleuchtung je Tag. Nach unseren Beobachtungen überschreitet es bei den vorliegenden Kulturbedingungen sicherlich 2500 Lux. Der Gegensatz darf darauf zurückgeführt werden, daß $\mathrm{D}$ a o die Algen in reinem Seewasser, also im Gegensatz zu uns in nährstoff-armem Milieu kultivierte und dementsprechend auch viel teratologische Bildungen beobachtete.

5 S. D a o, Mém. Fac. Sci. Paris, $\mathrm{N}^{\circ} 1325,1954$. 

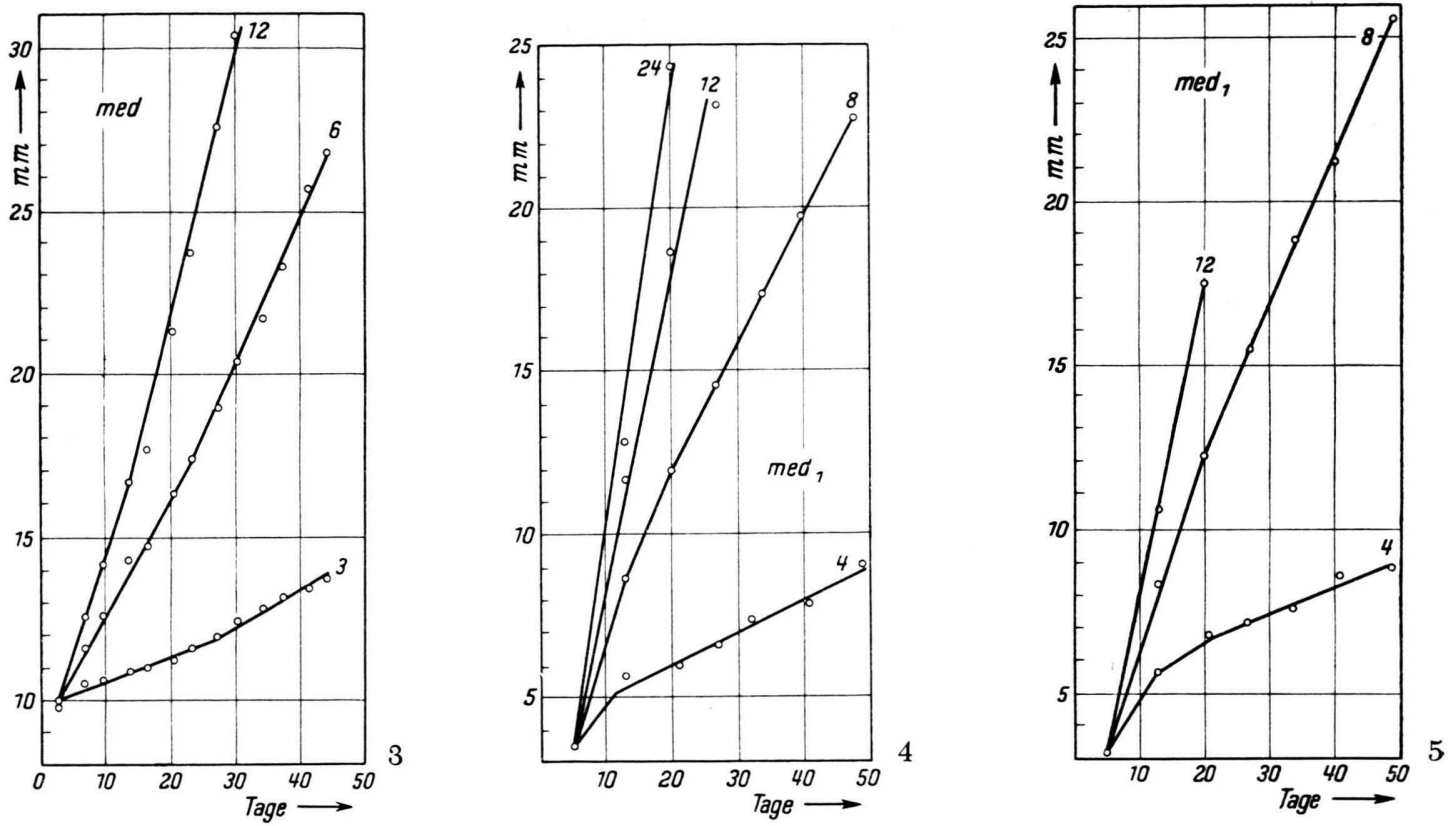

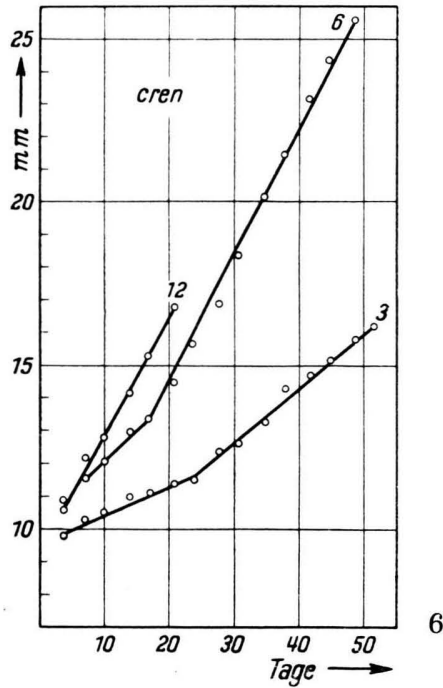

Abb. 3. A. mediterranea. Zunahme der Stiellänge (mm) unbehandelter Zellen. Tägliche Beleuchtungsdauer $12-$ $6-3$ Stunden. Normallicht. $21^{\circ}$

Abb. 4. A. mediterranea. Zunahme der Stiellänge regenerierender Zellen. Tägliche Beleuchtungsdauer (Normallicht) $24-12-8-4$ Stunden. $21^{\circ}$.

Abb. 5. A. mediterranea. Zunahme der Stiellänge (mm) regenerierender Zellen. Tägliche Beleuchtungsdauer (Normallicht) $12-8-4$ Stunden. $27^{\circ}$.
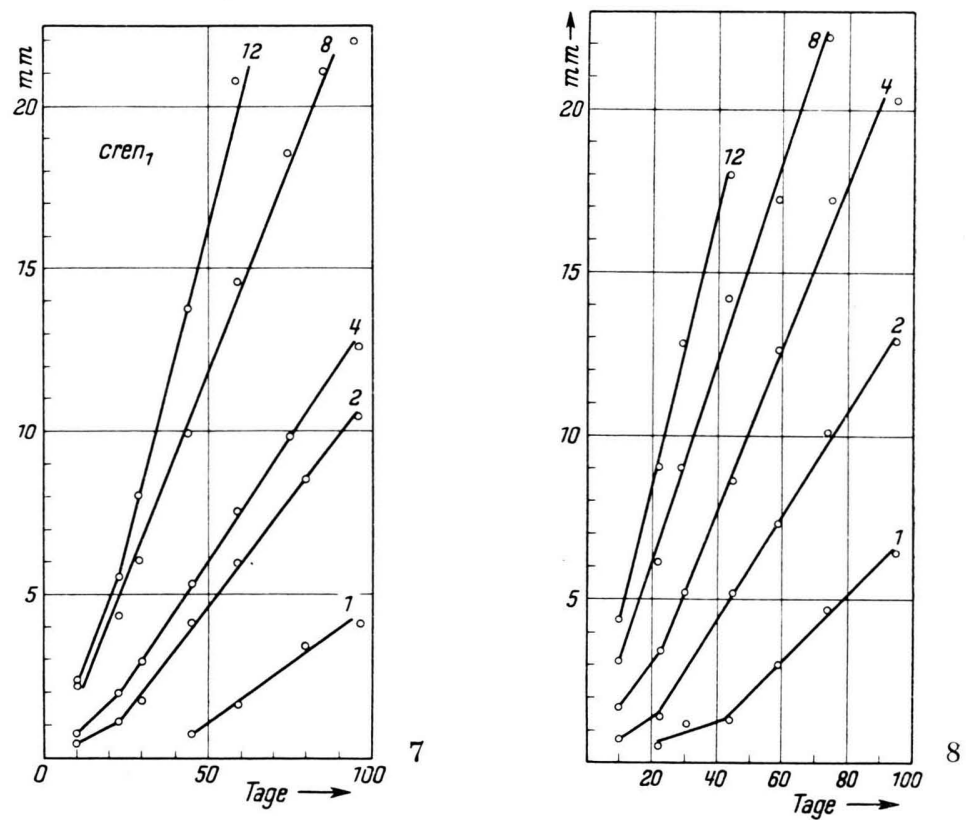

Abb. 6. A. crenulata. Zunahme der Stiellänge (mm) unbehandelter Zellen. Tägliche Beleuchtungsdauer (Normallicht) $12-6-3$ Stunden. $21^{\circ}$.

Abb. 7. A. crenulata. Zunahme der Stiellänge ( $\mathrm{mm})$ regenerierender Zellen. Tägliche Beleuchtungsdauer $12-8$ $-4-2-1$ Stunden. Normallicht alter Art (Glühlampe wassergekühlt, etwa $2500 \mathrm{Lux}) .21^{\circ}$.

Abb. 8. A. crenulata. Zunahme der Stiellänge (mm) regenerierender Zellen. Tägliche Beleuchtungsdauer $12-8$ $-4-2-1$ Stunden. Starklicht. $21^{\circ}$. 


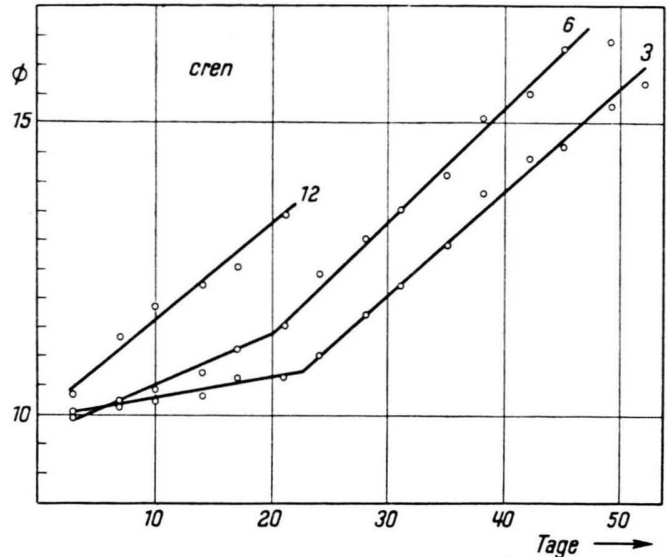

Abb. 9. A. crenulata. Zunahme des Stieldurchmessers unbehandelter Zellen. Tägliche Beleuchtungsdauer (Normallicht) $12-6-3$ Stunden. $21^{\circ}$. (Zunahme der Stiellänge vgl. Abb. 6.)

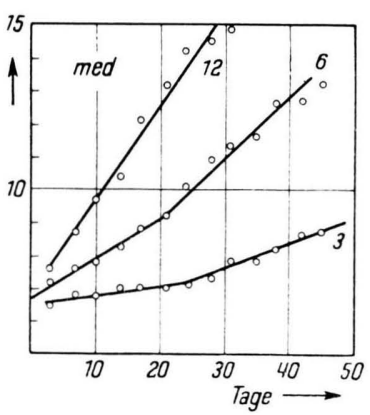

Abb. 10. A. mediterranea. Zunahme des Stieldurchmessers unbehandelter Zellen. Tägliche Beleuchtungsdauer (Normallicht) $12-6-3$ Stunden. $21^{\circ}$ (Zunahme der Stiellänge vgl. Abb. 3).

(Tab. 6, Sp. 4) zeigt etwa gleichen Zuwachs bei 3-, 6- und 12-stdg. Beleuchtung täglich und verhält sich somit anders als die beiden zuerst behandelten Versuchsreihen; allerdings nehmen die aus der Abb. 9 $\mathrm{zu}$ errechnenden Werte der Stieldurchmesser etwas $\mathrm{zu}$ vom 12 - über den 6 - zum $3 \mathrm{~h}$-Tag.

Die Zuwachswerte für A. mediterranea (Tab. 7, Sp. 2-4) lassen sich nicht in eine sinnvoll erscheirende Reihe wie die beiden crenulata-Serien bringen. Vielleicht folgen sie einer Optimumkurve, die ihr Maximum beim 8-12h-Tag hat. Dauerlicht wird auch hier schlechter verwertet als das Licht des $12 \mathrm{~h}$-Tages.

b) Beobachtungen über den Einfluß der Temperatur-Erhöhung von 21 auf $27^{\circ}$ auf das Stielwachstum liegen bei A.mediterranea (Abb. 4 und 5 und Tab. 7, Sp. 3 und 4) und A. crenulata (Tab. 8, Sp. 2 und 4) vor. Bei A. crenulata wird das Stielwachstum fast um das Doppelte beschleunigt. Bei A. medi-

\begin{tabular}{|c|c|c|c|c|}
\hline \multirow{2}{*}{$\begin{array}{c}\text { Tägliche } \\
\text { Beleuchtungsdauer } \\
{[\mathrm{h}]} \\
1\end{array}$} & \multicolumn{2}{|c|}{$\begin{array}{l}\text { Regenerierende } \\
\text { Zellen }\end{array}$} & \multicolumn{2}{|c|}{$\begin{array}{l}\text { Unbe- } \\
\text { handelte } \\
\text { Zellen }\end{array}$} \\
\hline & 2 & 3 & & \\
\hline 12 & 3,8 & 4,0 & 3,5 & - \\
\hline 8 & 3,9 & 4,5 & - & - \\
\hline 6 & - & - & 3,8 & 8,0 \\
\hline 4 & 6,2 & 7,5 & - & \\
\hline 3 & - & $\overline{0}$ & 3,6 & 7,2 \\
\hline $\begin{array}{l}2 \\
1\end{array}$ & $\begin{array}{l}6,9 \\
8,4\end{array}$ & $\begin{array}{r}9,0 \\
12,0\end{array}$ & - & Z \\
\hline & & & & \\
\hline
\end{tabular}

Tab. 6. A. crenulata. Zuwachs der Stiellängen $(\mathrm{mm})$ in 120 Lichtstunden (vgl. Text). 21 ${ }^{\circ}$. Sp. 2: vgl. Abb. 7. Normallicht etwa 2500 Lux (Glühlampe). Sp. 3: vgl. Abb. 8. Starklicht etwa 5000 Lux. Sp. 4: vgl. Abb. 6. Normallicht etwa $2500 \mathrm{Lux}$ (Leuchtstoff-Lampen).

\begin{tabular}{|c|c|c|c|}
\hline \multirow{2}{*}{$\begin{array}{c}\text { Tägliche } \\
\text { Beleuchtungsdauer } \\
{[\mathrm{h}]}\end{array}$} & \multicolumn{2}{|c|}{$\begin{array}{l}\text { Regenerierende } \\
\text { Zellen }\end{array}$} & \multirow{2}{*}{$\begin{array}{c}\text { Unbe- } \\
\text { handelte } \\
\text { Zellen } \\
21^{\circ}\end{array}$} \\
\hline & $21^{\circ}$ & $27^{\circ}$ & \\
\hline 1 & 2 & 3 & 4 \\
\hline 24 & $6.5-$ & -- & - \\
\hline 12 & $10,6 \quad 6,7$ & $9,6-$ & 5,9 \\
\hline 8 & $-5,4$ & $9,2 \quad 6,3$ & $-\quad-$ \\
\hline 6 & -- & -- & $8,0 \quad 10,0$ \\
\hline 4 & $-4,4$ & $12,0 \quad 2,4$ & - \\
\hline 3 & -- & -- & 2,4 \\
\hline
\end{tabular}

Tab. 7. A. mediterranea. Zuwachs der Stiellängen (mm) in 120 Lichtstunden (vgl. Text). Normallicht (LeuchtstoffLampen) 2500 Lux. Sp. 2: vgl. Abb. 4. Sp. 3: vgl. Abb. 5. Sp. 4: vgl. Abb. 3.

terranea ist die Erhöhung praktisch wirkungslos, weil offenbar das Temperaturoptimum schon überschritten ist (vgl. auch S. 273).

2. Über Lichtwirkung auf die Hutbildung wurde schon in einer früheren Arbeit berichtet ${ }^{2}$. Versuche an kernhaltigen Zellen zeigten bei mehreren Arten, daß für den Zeitpunkt der Hutbildung im Schwachlicht eine einfache Beziehung zur Lichtmenge besteht: eine bestimmte Gesamtlichtmenge, deren Größe u. a. artcharakteristisch ist, muß eingestrahlt worden sein, damit Hutbildung erfolgen kann. Bei höheren Lichtintensitäten wurden die Hutbildungszeiten nicht gemessen. Sicher ist, daß auch in diesen Bereichen Erhöhung der täglichen Lichtmenge einen früheren Zeitpunkt der Hutentstehung bewirkt, die Gesamtheit der vorbereitenden Hutbildungsprozesse also durch höheres Lichtangebot beschleunigt wird. 
Es erscheint allerdings fraglich, ob die quantitativen Beziehungen stets in einer einfachen Produktenregel wie beim Schwachlicht dargestellt werden können.

Hervorzuheben ist, daß die Hutbildung im Verhältnis $\mathrm{zu}$ anderen Prozessen überhaupt ein hohes Lichtbedürfnis hat. So bilden crenulata-Zellen bei $4 \mathrm{~h}$ Schwachlicht gar keine Hüte mehr, obwohl ihr Stiel noch ständig, wenn auch natürlich langsam, wächst ${ }^{2}$. Auch Beobachtungen über die Häufigkeit von Hutpflanzen im Vergleich zu hutlosen Zellen (vgl. folgende Arbeit) zeigen, daß die Verhältnisse für die Hutbildung mit steigender Beleuchtungsmenge günstiger werden.

Der Einfluß der Temperatur auf die Hutbildung geht u. a. aus Tab. 8 (vgl. Sp. 2 und 4) hervor. Temperaturerhöhung von 21 auf $27^{\circ}$ bewirkt in diesem Versuch nach 23 Tagen eine Erhöhung des Hutpflanzen-Anteils von 15 auf 100 Prozent. Auch alle anderen Beobachtungen lassen erkennen, daß diese Temperaturerhöhung Hüte beschleunigt entstehen läßt.

\section{III.}

Licht- und Temperaturerhöhung wirken, wie wir sahen, bis auf wenige Ausnahmen auf Stielwachstum und Hutbildung beschleunigend. Diese Aussage kann auf Grund der Befunde über die endgültigen Stiellängen noch erweitert werden. Würde nämlich der Ablauf der vorbereitenden Hutbildungs- und Stielwachstums-Prozesse durch Veränderungen der Außenfaktoren in gleichem $\mathrm{Maße}$ betroffen werden, so müßte man stets die gleiche endgültige Stiellänge feststellen. Dies ist, wie eingangs gezeigt wurde, jedoch nicht der Fall. Daraus folgt, daß beide Prozesse in verschiedenem Maße von Veränderungen der Außenfaktoren beeinflußt werden. Ferner bedeutet das Ergebnis, daß Stiellängen-Unterschiede verwertet werden können, um Aussagen über die Relation der Abhängigkeit beider Prozesse von Außenfaktoren zu machen.

Tab. 8 demonstriert die unterschiedliche Geschwindigkeit der Stielwachstums- und Hutbildungs-Prozesse bescnders deutlich sowie das Zusammenwirken beider, als deren Resultante die endgültige Stiellänge erscheint. Durch Erhöhung der täglichen Beleuchtungsdauer wird die Stielwachstums-Geschwindigkeit gar nicht mehr verändert, wohl aber die Hutbildung beschleunigt: die Hutpflanzen sind deswegen kürzer. Durch Temperaturerhöhung wird die StielwachstumsGeschwindigkeit offenbar mehr beschleunigt als die Hutbildung: die Hutpflanzen sind deswegen länger.

\begin{tabular}{|c|c|c|c|}
\hline 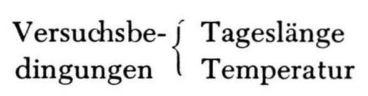 & $\begin{array}{l}12^{\mathrm{h}} \\
21^{\circ}\end{array}$ & $\begin{array}{l}24^{\mathrm{h}} \\
21^{\circ}\end{array}$ & $\begin{array}{l}12^{\mathrm{h}} \\
27^{\circ}\end{array}$ \\
\hline $\begin{array}{l}\text { Stiel- } \\
\text { länge }\left\{\begin{array}{l}\text { bei Versuchsbeginn } \\
10 \text { Tage darnach } \\
\text { [mm] }\end{array} 17 \text { Tage darnach }\right.\end{array}$ & $\begin{array}{r}0,0 \\
6,5 \\
11,7\end{array}$ & $\begin{array}{r}0,0 \\
6,8 \\
11,5\end{array}$ & $\begin{array}{r}0,0 \\
11,1 \\
19,7\end{array}$ \\
\hline Stiellänge bei 1 . Hut & 19,4 & 15,5 & 23,0 \\
\hline $\begin{array}{l}\text { Hutpflanzen }(\%) 23 \text { Tage } \\
\text { nach Versuchsbeginn }\end{array}$ & 15 & 86 & 100 \\
\hline$n$ & 27 & 22 & 13 \\
\hline
\end{tabular}

Tab. 8. Regenerierende Zellen von A.crenulata. Geschwindigkeit des Stielwachstums und Stiellänge bis 1. Hut.

Aus den Licht-Versuchen zur endgültigen Stiellänge kann man also folgern:

1. Erhöhung der täglichen Lichtmenge durch längere Beleuchtung oder Intensitätssteigerung beschleunigt die Hutbildung relativ mehr als das Stielwachstum. 2. Sowohl die optimale als auch die minimale Beleuchtungsmenge pro Tag ist für die Hutbildung offenbar größer als für das Stielwachstum. Das eine darf aus der Tatsache geschlossen werden, daß Tagesverlängerung von 12 auf $24 \mathrm{~h}$ nur noch Beschleunigung der Hutbildung bewirkt (Tab. 8, Sp. 2 und 3). Das andere geht aus den bereits angeführten früheren Versuchen hervor, wonach die vorbereitenden Hutbildungs-Prozesse im Gegensatz zum Stielwachstum bei geringem Lichtangebot nicht mehr vollständig ablaufen, so daß die Hutbildung ausfällt ${ }^{2}$.

Kurz zusammenfassend kann man sagen, daß die Gesamtheit der vorbereitenden Hutbildungs-Prozesse ein höheres Lichtbedürfnis hat als die Stielwachstums-Vorgänge.

In bezug auf die Temperatur lautet die Folgerung aus den Stiellängen-Unterschieden: Temperaturerhöhung von 21 auf $27^{\circ}$ beschleunigt bei Acicularia und A. crenulata das Stielwachstum, bei A. mediterranea dagegen die Hutbildung relativ stärker. Die Wachstums-Beobachtungen zeigten (S.272), daß Temperaturerhöhung bei A. crenulata das Stielwachstum noch fördert, bei A. mediterranea dagegen nicht mehr. Der Unterschied zwischen den Arten Acicularia und A. crenulata einerseits und A. mediterranea andererseits beruht also wesentlich auf dem Temperatureinfluß auf die Geschwindigkeit des Stielwachstums. Die Hutbildung wird bei allen drei Arten noch beschleunigt, ob vielleicht bei Acicularia und A. crenulata mehr als bei A. mediterranea, läßt sich auf 
Grund der bisherigen Versuche nicht sagen. A. mediterranea hat zum mindesten in bezug auf das Stielwachstum ein niedrigeres Temperaturoptimum als die beiden anderen Arten. Diese Tatsache steht in Einklang mit der natürlichen Verbreitung: A. mediterranea kommt in höheren Breiten vor als Acicularia und $A$. crenulata.

Auf den ersten Blick scheint der Befund, daß Erhöhung der Lichtmenge die Hutbildung, Erhöhung der Temperatur dagegen das Stielwachstum relativ mehr beschleunigt, eine andersartige Wirkung anzuzeigen. Dies braucht allerdings nicht der Fall zu sein. Man kann sich nämlich vorstellen, daß Hutbildung und Stielwachstum einer ähnlichen Optimumkurve folgen, wobei die Kurve der Hutbildung gegenüber

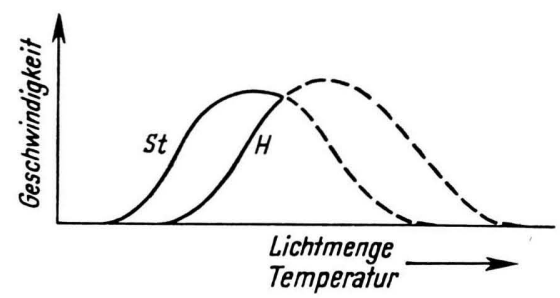

Abb. 11. Vgl. Text.

der Stielwachstumskurve parallel zur Ordinatenachse etwas nach rechts verschoben ist (vgl. Abb. 11). Bei dieser Vorstellung verhalten sich Licht und Temperatur grundsätzlich gleichartig. Man findet nämlich je nach der Steilheit der Kurven Abszissenbereiche, in denen die Hutbildung, wie auch solche, in denen das Stielwachstum relativ zum anderen Prozeß beschleunigt ist. Ist dies auch nicht die einzig mögliche Vorstellung, so fügen sich ihr doch alle bisher vorliegenden Befunde ein.

Besprechung der Versuchsergebnisse

I.

Die im Vorausgegangenen dargestellten Versuchsergebnisse haben die Verschiedenheit der Stielwachstums-Vorgänge und der gleichzeitig ablaufenden vorbereitenden Hutbildungs-Prozesse an ihrer unterschiedlichen Abhängigkeit von Außenfaktoren und ihrem Einfluß auf die endgültige Stiellänge erkennen lassen. Es fragt sich, welche Beziehungen zwischen beiden Vorgängen bestehen.

Zunächst ist auf folgendes hinzuweisen. Beim Stielwachstum, also dem Weiterwachsen des Stieles, denkt man vor allem an die Substanzvermehrung eines vorhandenen Gebildes, die Hutbildung dagegen

6 K. B e th, Z. Naturforschg. 8 b, 771 [1953]. ist ein neuer, andersartiger Differenzierungs-Vorgang, und zwar ein komplizierter Vorgang, der sich an dem einfach gebauten Stiel vollzieht. Jedoch muß schon die Anlage eines Hutes auch als komplizierter Wachstumsvorgang angesehen werden (Zellwand!), außerdem wächst der Hut nach seiner Anlage unter Substanzvermehrung aus. Umgekehrt ist auch das Weiterwachsen des Stieles natürlich ein DifferenzierungsProzeß. Beide Vorgänge, Stiel- und Hutentstehung, können sowohl vom Wachstum wie auch von der Differenzierung her betrachtet werden. Die Formulierung - während des Stielwachstums finden vorbereitende Hutbildungs-Prozesse statt - betont beim Stiel den Wachstums-, beim Hut den Differenzierungs-Vorgang. Man sollte besser sagen: während einer bestimmten Differenzierungsform (Stielbildung) werden Voraussetzungen für eine andere Differenzierungsform (Hutbildung) geschaffen.

Von den Befunden sei hierzu die morphologischentwicklungsgeschichtliche Tatsache angeführt, daß kein Fall von Hutbildung ohne vorhergegangenes Stielwachstum bekannt ist. Das apikale Wachstum der Acetabularia-Zelle erfolgt immer erst in der Form des Stielwachstums, erst auf späteren Entwicklungsstadien wird es von Hutbildung und -wachstum abgelöst und kann nach diesem auch wieder einsetzen. Hiernach stellt das Stielwachstum eine notwendige Voraussetzung der Hutbildung dar und das Stielwachstum erscheint als eine „niedere“, das Hutwachstum als eine „höhere“ Form des Wachstums.

Betrachtet man die physiologischen Unterschiede, so fällt zunächst eine gewisse Unabhängigkeit beider Vorgänge auf. Die gleitenden Verhältnisse bei den endgültigen Stiellängen zeigen, daß sich beide Vorgänge in ihrem Geschwindigkeitsablauf offenbar unschwer gegeneinander verschieben lassen. Eine Verschiebung ist auch bei der verfrühten Hutbildung kernloser junger Entwicklungsstadien zu erkennen, die in diesem Fall sogar sehr schnell erfolgt ${ }^{6}$. Natürlich darf daraus nicht geschlossen werden, daß beide Vorgänge völlig unabhängig voneinander sind. Es sei hier an die Unregelmäßigkeit im Stielzuwachs erinnert, die angesichts der Gültigkeit der StiellängenRegel von ähnlichen Unregelmäßigkeiten im Ablauf der Hutbildungs-Prozesse begleitet gewesen sein muß (S. 270, 272). Dieser Befund läßt an irgendeine Art von Koppelung oder gemeinsame Wurzel beider Vorgänge denken. In diesem Zusammenhang sei erwähnt, daß Brachet und Brygier ${ }^{7}$ auf Grund

7 J. Brachet u. Brygier, Arch. internal Physiol. 61, 246 [1953]. 
ihrer Befunde über den stark licht-abhängigen Einbau von radioaktivem $\mathrm{CO}_{2}$ im Gegensatz zum kaum vom Licht beeinflußten Einbau von Glykokoll meinen, daß es bei Acetabularia verschiedene Mechanismen der Eiweißsynthese mit unterschiedlicher Lichtempfindlichkeit geben könnte. Man kann sich aber wohl auch vorstellen, Hutbildung und -wachstum wären u. a. durch Bildung anderer Eiweiße im Vergleich zu denen der Stiele charakterisiert und jene könnten nur aus diesen entstehen. Mit dieser Vorstellung würden alle bisher bekannten Unterschiede zwischen Bildung und Wachstum von Stiel und Hut in Einklang $\mathrm{zu}$ bringen sein. Es würden die entwicklungsgeschichtlichen Beziehungen plausibel, es ließen sich die oben erwähnten gleitenden Verhältnisse und die lange Hutvorbereitungszeit einordnen; es würde die mit Hutbildung und -wachstum verbundene Atmungssteigerung ${ }^{8}$ und vor allem der für das Eintreten der Hutbildung notwendige hohe Energiebedarf verständlich.

\section{II.}

Die bisherigen Aussagen können noch etwas erweitert werden. Eingangs wurde schon gesagt, daß sich bei den vorbereitenden Hutbildungs-Prozessen kern-abhängige und kern-unabhängige Vorgänge unterscheiden lassen. Die kern-abhängigen können ebenso wie die gesamten, zum Stielwachstum gehörigen Vorgänge bei niedrigen Lichtintensitäten ablaufen, dagegen verlangen die kern-unabhängigen hut-bildenden Prozesse höhere Lichtgaben. Dieser charakteristische Unterschied zwischen Stiel- und Hutbildungs-Prozessen beruht also auf Verschiedenheiten im Energiebedarf der cytoplasmatischen Vorgänge, die nicht mehr unmittelbar kern-abhängig sind. Das gleiche gilt nach den hier dargestellten Versuchen auch für den breiten Bereich, in dem Stiel- und Hutbildung vollständig ablaufen können. Die kernunabhängigen Vorgänge des Cytoplasmas sind also auch für die hier diskutierten Befunde von besonderer Bedeutung.

Die große Bedeutung des Cytoplasmas für die vorbereitenden Hutbildungs-Prozesse ist noch aus zwei anderen Befunden deutlich zu erkennen. Erstens wird nach Versuchen an kernlosen Teilen (vgl.l.c. ${ }^{2}$ S. 337) die Geschwindigkeit der kern-unabhängigen

8 M. B. Chantrenne-van Halteren u. J. B r a chet, Arch. internal Physiol. 60, 187 [1952]; J. Brachet u. Brygier, Arch. internal Physiol. 61, 246 [1952].

9 Vorlesungen über Entwicklungsphysiologie, Springer 1954.
Vorgänge wesentlich von der Höhe der eingestrahlten Energie bestimmt. Zweitens ist die Geschwindigkeit der Hutbildung bei kern-haltigen Pflanzen ebenfalls abhängig von der eingestrahlten Energie, aber unabhängig davon, ob der Kernbezirk verdunkelt ist oder nicht (1. c. ${ }^{2}$ S. 336).

Wenn sich in den erwähnten früheren Versuchen in bezug auf die Hutbildung ein Einfluß der eingestrahlten Energiemenge auf die Kernleistung erkennen ließ und betont wurde, daß die kern-unabhängigen Folgeprozesse den vorausgegangenen $\mathrm{Ab}$ lauf der kern-abhängigen Prozesse zur Voraussetzung haben, der Kern also von primärer Bedeutung ist, so soll hier darauf hingewiesen werden, daß es dennoch möglich ist, die Gesamtheit der Vorgänge in der kern-haltigen Zelle als entscheidend vom Cytoplasma bestimmt anzusehen. Bei der kern-haltigen Pflanze wird nämlich mit einem dynamischen Gleichgewicht zwischen allen Vorgängen zu rechnen sein, und man kann sich unschwer vorstellen, daß hinsichtlich der vorbereitenden Hutbildungs-Prozesse durch eine Beschleunigung der kern-unabhängigen Prozesse als Folge erhöhter Photosynthese dieses Gleichgewicht $\mathrm{zu}$ ihren Gunsten verschoben wird. Dies müßte einen beschleunigten Ablauf der kernabhängigen Prozesse zur Folge haben und dies wiederum den Kern zu erhöhter Tätigkeit veranlassen. Unter der bestimmenden Rolle des Cytoplasmas soll also nicht etwa nur verstanden werden, daß die unmittelbare Energieaufnahme und -umwandlung vom Kern unabhängig ist und der Kern die für seine Tätigkeit erforderliche Energie vom Cytoplasma bezieht, sondern es ist damit noch die Abhängigkeit der Kernwirkungsstärke vom Cytoplasma in der eben skizzierten Art gemeint (vgl. a. $\mathrm{K} \ddot{\mathrm{u} h \mathrm{n}^{9}}{ }^{\mathrm{S}}$ S. $483 \mathrm{ff}$.).

Die erwähnte Vorstellung steht zudem in Einklang mit denjenigen Versuchen an Acetabularia, welche eine Parallelität zwischen dem Stielwachstum und der Kernentwicklung ergaben, wobei der für beide Vorgänge übergeordnete Faktor der jeweils mögliche cytoplasmatische Energiegewinn ist ${ }^{10}$. Die aus den Versuchen zur Hutbildung ableitbare Folgerung, daß der Kern je nach seinem von der cytoplasmatischen Energieproduktion gesteuerten Zustande unterschiedliche Wirkungsstärken besitzt, bestätigte sich in speziellen Versuchen ${ }^{11}$. Mindestens ein Teil der intra-

10 H. Stich, Z. Naturforschg. 6 b, 319 [1951]; Z. Naturforschg. 8 b, 36 [1953]; Chromosoma, im Druck; J. B r a c h e t, Experientia 8, 347 [1952]; Colston Papers VII, 91, London 1954.

$11 \mathrm{~J}$. H ä m m e r li ng, 8me Congr. Intern. de Botan. Paris 1954, im Druck; G. W e r z, Planta, im Druck. 
und extranukleären Prozesse sind offenbar in Form von Kreisprozessen miteinander gekoppelt und in ihrer Geschwindigkeit von der photosynthetischen Leistung abhängig (vgl. a. l. c. ${ }^{12}$ ).

\section{III.}

Im Mittelpunkt unserer Betrachtungen stehen Erscheinungen, die bei vielen Metaphyten oft untersucht worden sind, z. B. das höhere Lichtbedürfnis der reproduktiven Phase sowie die etiolements-verhindernde Wirkung des Lichtes.

Die hier geschilderten Beobachtungen sind deshalb von entwicklungsphysiologischem Interesse, weil sie an einem einzelligen Organismus Zusammenhänge erkennen lassen, die bei vielzelligen Pflanzen nicht möglich sind. So erscheint die Stiellänge als Resultante zweier von Außenfaktoren verschieden beeinflußbarer Vorgänge. Ferner erlaubt die Gunst des
Objektes, kern-abhängige und kern-unabhängige Teile dieser Vorgänge zu unterscheiden. Bedeutungsvoll ist außerdem, daß wir hier ein Entwicklungsstadium der Einzelzelle untersuchen können, das von Kern- oder gar Zellteilungen unbeeinflußt ist.

Für eine Beurteilung der gesamten Zellentwicklung muß auch die spätere, von den KernteilungsVorgängen beeinflußte Phase berücksichtigt werden. Entsprechend den hier dargestellten Beziehungen zwischen Stielwachstums- und vorbereitenden Hutbildungs-Prozessen gibt es Befunde, welche Beziehungen zwischen diesen beiden und den KernteilungsVorgängen und das gemeinsame Wirken aller drei Prozesse erkennen lassen. Diese sollen in der folgenden Arbeit mitgeteilt werden.

$12 \mathrm{~J}$. H ämmerling, Exp. Cell Res., im Druck; C. H. W a d ding t o n, Colston Papers VII, 105, London 1954.

\title{
Unterschiedliche Beeinflussung von Wachstum und Teilung durch Veränderung von Licht und Temperatur*
}

\author{
Von Kurt Beth \\ Aus dem Max-Planck-Institut für Meeresbiologie, Abt. Hämmerling, Wilhelmshaven \\ (Z. Naturforschg. 10 b, 276-281 [1955]; eingegangen am 7. Februar 1955)
}

\begin{abstract}
Die Zellen von Acicularia (Acetabularioideae) werden kleiner, wenn sie täglich mehr Licht erhalten, sie werden großer, wenn sie statt bei 21 bei $27^{\circ}$ kultiviert werden. Die ZellgrößenUnterschiede lassen relative Unterschiede in den Geschwindigkeiten erkennen, mit der die für Stielwachstum, Hutbildung und Kernteilung erforderlichen Vorgänge ablaufen. Von diesen Vorgängen haben die Hutbildungsprozesse das höchste Lichtbedürfnis. Dies zeigen auch Beobachtungen bei Acicularia und Acetabularia polyphysoides über das Verhältnis von Cystenpflanzen mit und ohne Hut, das sich bei geringer werdendem Lichtangebot zugunsten der hutlosen Cystenpflanzen verschiebt.
\end{abstract}

1. Nach Licht- und Temperaturversuchen an mehreren Acetabularia-Arten können Verschiedenheiten in den Stiellängen der Zelle im Augenblick der Hutbildung durch unterschiedliche Beschleunigung von Stielwachstums- und vorbereitenden Hutbildungsprozessen erklärt werden ${ }^{1}$. Im Folgenden soll die Wirkung beider Außenfaktoren auf einen dritten Prozeß, nämlich die Kernteilung, dargestellt werden.

2. Die für Acetabularia charakteristischen Wachstums- und Formbildungsvorgänge (Rhizoid-, StielWirtel-, Hutbildung) spielen sich an einer einkernigen Zelle ab und finden zwischen der Zygotenbildung und der ersten Kernteilung statt. Erst mehrere

1 K. B e th, Z. Naturforschg. 10 b, 267 [1955].
Monate nach der Zygotenkeimung bildet der Primärkern in schneller Folge viele Sekundärkerne, welche durch eine Plasmaströmung zusammen mit den Chloroplasten in den Hut transportiert werden, wo sich um jeden Kern eine Cyste bildet. Zwischen der ersten Primärkernteilung und der Cystenbildung vergehen nur wenige Tage. Abgesehen von der durch den Chloroplastentransport bedingten Stielaufhellung stellt also die Cystenbildung den makroskopisch sichtbaren Ausdruck der kurz zuvor erfolgten Kernteilung dar. Normale Cystenbildung kann bei ver-

\footnotetext{
* Mit Unterstützung der Deutschen Forschungs g e meins chaf $\mathrm{t}$ für die in dieser Arbeit enthaltenen Probleme seit 1.4. 1953.
} 
schiedenen Arten, so auch bei der hier untersuchten Acicularia und Acetabularia polyphysoides nicht nur im Hut stattfinden, sondern auch im Stiel, einerlei ob ein Hut vorhanden ist oder nicht. Der Hut stellt also bei diesen Arten keine notwendige Voraussetzung zur Kernteilung und deren Folgeprozessen bis zur Cystenbildung dar (Einzelheiten vgl. ${ }^{2,3}$ ).

3. Die Zelle hat ihre maximale Größe kurz vor der Kernteilung erreicht. Die endgültige Zellgröße ist in regelmäßiger Weise abgeändert, wenn die Zellen bei verschiedenen Lichtmengen oder verschiedener Temperatur kultiviert werden. Bei hutlosen Cystenpflanzen kann die Änderung der Zellgröße infolge abgestufter Außenbedingungen unmittelbar aus der endgültigen Länge des Stieles, dessen Durchmesser

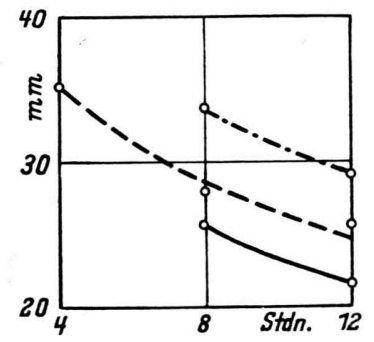

Abb. 1. Acicularia schenckii. Stiellänge hutloser Cystenpflanzen in Abhängigkeit von Temperatur und täglich gebotener Lichtmenge (Tageslänge und Intensität).

$-21^{\circ}$ Normallicht, - - - - $27^{\circ}$ Normallicht, ....... $21^{\circ}$ Schwachlicht.

nicht wesentlich verändert ist, abgelesen werden. Abb. 1 (vgl. oberste und unterste Kurve) zeigt für Acicularia schenckii, daß die hutlosen Cystenpflanzen kürzer sind, wenn sie während der Aufzucht täglich mehr Licht erhalten haben, sei es durch längere Beleuchtungszeit bei einer gegebenen Lichtintensität, sei es durch Intensitätserhöhung bei einer gegebenen Beleuchtungszeit. Bei Temperaturerhöhung finden wir dagegen das umgekehrte Verhältnis: die ausgewachsenen Zellen sind bei $27^{\circ}$ länger als bei $21^{\circ}$ unter sonst gleichen Bedingungen (vgl. Abb. 1 mittlere und untere Kurve).

Die gleichen Beziehungen gelten für Zellen, die vor der Kernteilung Hüte gebildet haben. Die Zellgröße dieser Pflanzen ist im wesentlichen die Summe von Stiel- und Hutgröße. (Die Rhizoidgröße zeigt augenscheinlich keine nennenswerten Unterschiede.) Für die endgültigen Stiellängen von Hutpflanzen

2 J. H ä m m e r l in g, Biol. Zbl. 59, 158 [1939].

3 J. H ä m m e r l in g, Biol. Zbl. 51, 633 [1931]; K. L. S c h u l z e, Arch. Protistenkunde 92, 179 [1939];

\begin{tabular}{|c|c|c|c|c|c|c|c|c|c|}
\hline \multirow{2}{*}{$\begin{array}{c}\text { Licht } \\
\text { Temperat. }\end{array}$} & \multirow{2}{*}{$\begin{array}{c}\text { Tägliche } \\
\text { Beleucht.- } \\
\text { dauer } \\
{[\mathrm{h}]}\end{array}$} & \multicolumn{4}{|c|}{ I } & \multicolumn{4}{|c|}{ II } \\
\hline & & $M$ & $3 m$ & $\phi$ & $n$ & $M$ & $3 m$ & $\phi$ & $n$ \\
\hline Normal- & 24 & 36,8 & 1,7 & 4,3 & 60 & 41,6 & $\mid 1,0$ & 5,8 & 319 \\
\hline $\begin{array}{l}\text { licht } \\
21^{\circ}\end{array}$ & $\begin{array}{r}12 \\
8\end{array}$ & $\begin{array}{l}36,9 \\
38,3\end{array}$ & $\begin{array}{l}1,7 \\
3,2\end{array}$ & $\begin{array}{l}5,8 \\
7,0\end{array}$ & $\begin{array}{r}116 \\
48\end{array}$ & 41,2 & 1,4 & 6,5 & 192 \\
\hline $\begin{array}{l}\text { Normal- } \\
\text { licht } \\
27^{\circ}\end{array}$ & $\begin{array}{r}12 \\
8\end{array}$ & $\begin{array}{l}39,6 \\
37,3\end{array}$ & $\left|\begin{array}{l}1,5 \\
2,7\end{array}\right|$ & $\begin{array}{l}5,4 \\
6,0\end{array}$ & $\begin{array}{r}124 \\
52\end{array}$ & 43,5 & 1,7 & 5,8 & 123 \\
\hline $\begin{array}{c}\text { Schwach- } \\
\text { licht } \\
21^{\circ}\end{array}$ & 12 & 42,1 & 3,7 & 9,6 & 66 & - & - & - & - \\
\hline
\end{tabular}

Tab. 1. Acicularia schenckii. Durchmesser typischer Hüte $(1 / 10 \mathrm{~mm})$.

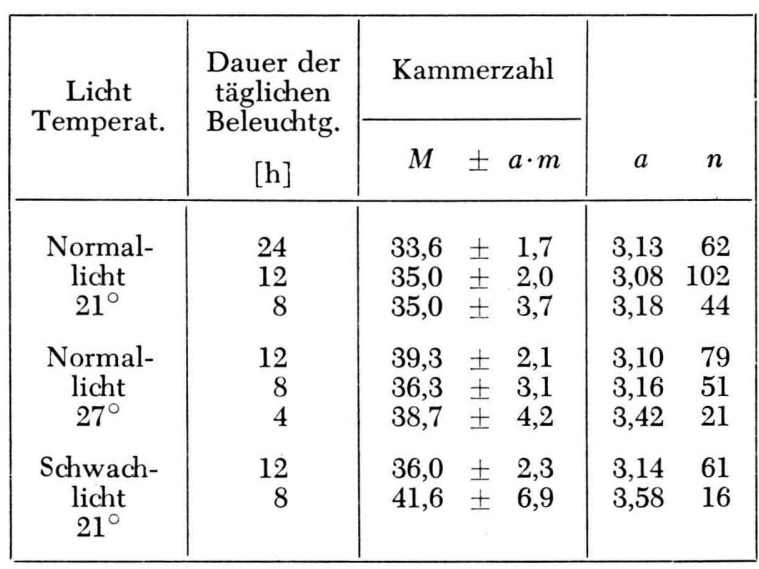

Tab. 2. Acicularia schenckii. Kammerzahl typischer Cystenhüte.

wurden schon in der vorigen Arbeit ${ }^{1}$ die bestehenden Unterschiede in Abhängigkeit von Licht und Temperatur dargestellt. Die Durchmesser- und Kammerzahlen von Acicularia-Hüten zeigen, daß der sich in der Veränderung der Stiellänge ausprägende Effekt nicht durch gegensinnige Abänderungen der Hutgröße kompensiert wird, sondern sogar etwas verstärkt sein kann. Denn ausgewachsene Zellen haben bei Faktorenänderungen, welche Stielverkürzung bewirken (mehr Licht, geringere Temperatur), keine Hüte mit größerem Durchmesser oder vermehrter Kammerzahl, sondern die Hüte sind z.T. etwas kleiner und haben z.T. etwas weniger Kammern (Tab. 1 und 2, vgl. besonders $12 \mathrm{~h}$ Normallicht 21 und $27^{\circ}$ ). Unterschiede in den Stiellängen

H. M a s c h l a n k a, Naturwissenschaften 31, 548 [1943]; J. Hä m m er ling, Arch. Protistenkunde 97, 7 [1944]. 


\begin{tabular}{|c|r|r|r|r|}
\hline $\begin{array}{c}\text { Licht } \\
\text { Temperatur }\end{array}$ & $\begin{array}{c}\text { Tages- } \\
\text { länge } \\
{[\mathrm{h}]}\end{array}$ & $\begin{array}{c}\text { Mit Hut } \\
{[\%]}\end{array}$ & $\begin{array}{c}\text { Ohne Hut } \\
{[\%]}\end{array}$ & $n$ \\
\hline \begin{tabular}{c|c|c|} 
Normallicht \\
$21^{\circ}$
\end{tabular} & 24 & 100 & 0 & 61 \\
& 12 & 89 & 11 & 157 \\
& 8 & 52 & 48 & 144 \\
Normallicht & 12 & 98 & 2 & 174 \\
$27^{\circ}$ & 8 & 77 & 23 & 102 \\
& 4 & 57 & 43 & 47 \\
Schwachlicht & 12 & 55 & 45 & 167 \\
$21^{\circ}$ & 8 & 31 & 69 & 65 \\
\hline
\end{tabular}

Tab. 3. Acicularia schenckii. Verhältnis hut-haltiger und hut-loser Cystenpflanzen.

können also gleichartigen Unterschieden in der Hutgröße entsprechen und sind Ausdruck der veränderten Zellgröße.

4. Können infolge ungünstiger Bedingungen keine Hüte gebildet werden, so wächst der Stiel, bis die Kernteilung erfolgt. Die Stiellänge bei hutlosen Cystenpflanzen wird durch die Geschwindigkeit des Stielwachstums und den Zeitpunkt der Kernteilung bestimmt. Würde Verstärkung eines Außenfaktors beide Vorgänge in gleichem Maße beeinflussen, so müßte die Stiellänge solcher Zellen gleich sein. Längenunterschiede von hutlosen Cystenpflanzen bei verschiedener Intensität eines Außenfaktors zeigen also dessen unterschiedliche Wirkung auf die beiden hier in Frage kommenden Vorgänge, und zwar werden durch Erhöhung der täglichen Lichtmenge die zur Kernteilung erforderlichen Prozesse relativ mehr gefördert, durch Temperaturerhöhung wird dagegen das Stielwachstum relativ mehr beschleunigt. Bei hut-bildenden Pflanzen wird durch Lichtverstärkung oder Temperaturerhöhung natürlich nicht nur das Stielwachstum, sondern auch das Hutwachstum gesteigert. Da bei Lichtverstärkung trotzdem bei Eintreten der Kernteilung nicht nur die Stiele, sondern in einigen Fällen auch die Hüte etwas kleiner sind, jedenfalls in keinem Fall größer, so gilt die angeführte Regel auch für Hutpflanzen.

5. Wenn bei erhöhten Lichtgaben sowohl die Hutbildung (siehe vorst. Arb. und Abb. 2) als die zur Kernteilung führenden Prozesse stärker beschleunigt werden als die Wachstumsprozesse in Stiel und Hut, so fragt sich, ob die Beschleunigung von Hutbildung und Kernteilung gleich groß ist. Hierüber geben die folgenden Befunde an Acicularia und Acetabularia polyphysoides Auskunft. Auch in hutlos bleibenden

\begin{tabular}{|c|c|c|c|}
\hline \multirow{2}{*}{$\begin{array}{l}\text { Tägliche } \\
\text { Beleuchtungs- } \\
\text { dauer } \\
{[\mathrm{h}]}\end{array}$} & \multicolumn{2}{|c|}{ Cystenpflanzen } & \multirow{2}{*}{$\begin{array}{c}\text { Anteil der } \\
\text { cystenlosen } \\
\text { Cy.-Pfl. } \\
{[\% / 0]}\end{array}$} \\
\hline & mit Hut & ohne Hut & \\
\hline 8 & 18 & 21 & 54 \\
\hline 11 & 40 & 34 & 46 \\
\hline 13 & 79 & 17 & 18 \\
\hline 16 & 72 & 7 & 9 \\
\hline 20 & 99 & 9 & 8 \\
\hline
\end{tabular}

Tab. 4. Cystenpflanzen mit und ohne Hut bei Polyphysa polyphysoides. Schwachlicht (etwa $600 \mathrm{Lux}, 21^{\circ}$ ).

Cystenpflanzen laufen vorbereitende HutbildungsProzesse ab, jedoch nicht vollkommen. Bei bestimmten Bedingungen findet man beide Zellsorten (Tab. 3 und 4). Bei manchen Zellen ist also der Ablauf der zur Hutbildung erforderlichen Prozesse, bei anderen Zellen der zur Kernteilung erforderlichen relativ mehr beschleunigt, so daß es dementsprechend zur Hutbildung kommt oder nicht. Vergleicht man die Verhältniszahlen bei den verschiedenen Tageslängen, so zeigt sich, daß bei höherem Lichtangebot relativ mehr Hüte entstehen, der vollständige Ablauf der zur Hutbildung führenden Prozesse gegenüber den zur Kernteilung erforderlichen also relativ mehr beschleunigt wird. Dies ist zunächst eine Bestätigung der früher geschilderten Befunde, wonach für die Hutbildung relativ hohe Lichtdosen erforderlich sind. Hinsichtlich der hier gestellten Frage ergibt sich, daß das Lichtbedürfnis der Kernteilung zwischen dem niedrigen des Stielwachstums und dem hohen der Hutbildung steht.

In einer früheren Arbeit ${ }^{4}$ wurde gezeigt, daß bei A. crenulata bei sehr geringer Lichtzufuhr der Stiel kontinuierlich, wenn auch natürlich sehr langsam wächst, aber weder Hutbildung noch Kernteilung erfolgt, obwohl die Stiellänge mit z. T. 10-12 cm wesentlich übersteigert war. Das darf man nach den hier geschilderten Befunden auch erwarten. Es ist hierbei allerdings zu berücksichtigen - und dies gilt auch für die schematische Darstellung der Versuchsergebnisse Abb. 2 -, daß nicht allein die Gesamtlichtmenge entscheidend ist. Die LichtmengenRegel gilt sicher nicht in allen Bereichen und unter allen Umständen (vgl. vorst. Arb.). Zu beachten ist u. a. noch der Intensitätsfaktor (oder vielleicht die täglich gebotene Lichtmenge). Denn bei A. crenulata findet unter einer bestimmten Lichtintensität bzw. täglich gebotenen Lichtmenge (600 Lux $4 \mathrm{~h}$ täglich)

\footnotetext{
${ }^{4}$ K. B e th, Z. Naturforschg. 8 b, 334 [1953].
} 


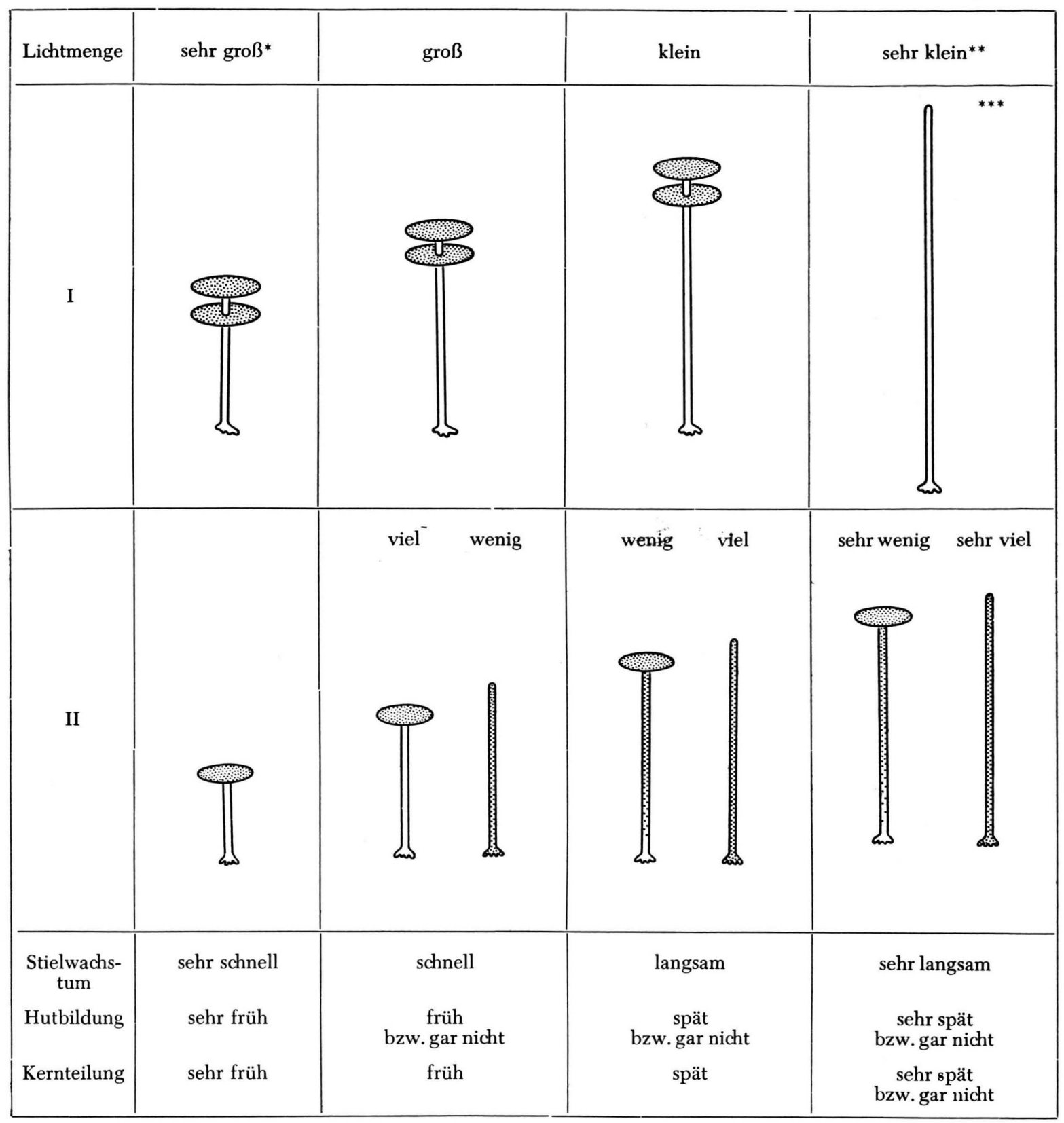

* Z. B. 2500 Lux Dauerlicht. $\quad{ }^{* *}$ Z. B. 600 Lux 4 h Tag. $\quad * * *$ Nur bei A. crenulata beobachtet, vgl. Text S. 278.

Abb. 2. Lichtmenge und Zellgröße. Schemat. Darstellung der Wirkung unterschiedlicher Lichtmengen auf Stielwachstum, Hutbildung und Kernteilung nach Versuchen an Acet. crenulata (I), die nur äußerst selten hutlose Cystenpflanzen bildet (ähnlich verhält sich Acet. mediterranea) und an Acicularia (II), die häufig hutlose Cystenpflanzen bilden kann (ähnlich verhalten sich Acet. polyphysoides und Acet. wettsteinii).

selbst bei jahrelanger Versuchsdauer keine Hutbildung mehr statt, obwohl die bei höheren Intensitäten erforderliche Gesamtlichtmenge weit überschritten ist. Es liegt kein Grund vor, für die KernteilungsVorgänge ein grundsätzlich anderes Verhalten in dieser Hinsicht anzunehmen. Es sei noch erwähnt, daß solche Systeme Ausfall der Hutbildung und Kernteilung nicht etwa wegen allgemeiner pathologischer Störung zeigen, sondern durch entsprechende Erhöhung der Intensität bei ausreichender Licht- 
menge jederzeit zu normaler Hutbildung und Kernteilung gebracht werden können.

6. Hinsichtlich der Temperaturwirkung auf die Zellgröße der hier untersuchten Arten ist zu sagen, daß eine Erhöhung von 21 auf $27^{\circ}$ eine Zellvergrößerung bewirkt, also einen der Lichtverstärkung entgegengesetzten Effekt hervorruft. Dies entspricht den meisten, wenn auch nicht allen Beobachtungen über die endgültige Stiellänge und das Verhältnis der Stielwachstums- und Hutbildungs-Geschwindigkeiten. Man möchte hierin zunächst eine grundsätzlich andersartige Wirkung von Licht und Temperatur sehen. Dies schließt nicht aus, daß sich die bisherigen Versuchsergebnisse mit der einfachen Annahme vereinbaren lassen, daß bei graphischer Darstellung der Wirkung von Licht- und Temperaturerhöhung das Kurvenbild grundsätzlich ähnlich sein kann (vgl. 1. c. ${ }^{1}$, Abb. 11). Versuche mit mehr und engeren Temperaturintervallen sind erforderlich, um Genaueres auszusagen. In dem schematischen Überblick der Versuchsergebnisse (Abb. 2) haben wir uns deshalb auf die einheitlichen Lichtwirkungen beschränkt.

7. $\mathrm{Zu}$ den hier geschilderten Beobachtungen kann ein Ergebnis an Eudorina unmittelbar in Beziehung gesetzt werden. Bei dieser Phytomonadine wird durch Dauerbelichtung die Teilung gegenüber dem Wachstum so stark gefördert, daß die Kolonien sich zu Tode teilen ${ }^{5}$. Umgekehrt hat $\mathrm{K} \mathrm{le} \mathrm{b} \mathrm{s}{ }^{6}$ gezeigt, daß (ähnlich wie bei A. crenulata) bei Farnprothallien im Schwachlicht keine Zellteilung, wohl aber ZellvergröBerung erfolgt. Auch die Untersuchungen an Ödogonium können hier erwähnt werden ${ }^{7}$. Im übrigen gibt es zwar sehr viele Beobachtungen, welche die Verschiedenartigkeit von Wachstums- und Teilungsprozessen auf Grund einer andersartigen Abhängigkeit von Außenfaktoren zeigen, das Augenmerk aber ist vorwiegend auf jeweils einen einzigen Prozeß und nicht auf das Zusammenspiel mehrerer Prozesse gerichtet gewesen. Die in dieser und der vorst. Arbeit geschilderten Versuchsergebnisse bestätigen nicht nur die an vielen pflanzlichen Objekten gefundene Regel über geringere Zellgrößen infolge Lichtverstärkung oder Temperaturerniedrigung (vgl. z. B. ${ }^{8}$ ), sondern zeigen darüber hinaus das Zusammenspiel dreier Zellprozesse: des Stielwachstums, der Hutbildung

5 M. H a rtmann, Arch. Protistenkunde 43, 223 [1921].

6 Sitzgsber. Heidelb. Akad. Wiss. mathem.-naturw. Kl. (1916, 1917).

7 H. F r e u n d, Planta 5, 520 [1928].

8 E. B ünning, Lehrb. Pflanzenphysiologie 2-3, 3. Aufl., Springer 1953.
(= Auslösung eines neuen Formbildungsvorganges) und der Kernteilung. Diese Prozesse werden durch Änderung eines Außenfaktors in gleicher Weise, aber unterschiedlichem Maße gefördert oder gehemmt, wobei die Unterschiede in Beschleunigung oder Hemmung der drei Prozesse wichtig für das Verständnis der Zellgrößen-Änderung sind. Der Einfluß des Lichtes auf die Zellgröße darf wohl im wesentlichen auf die Verhältnisse zwischen der photosynthetisch gewonnenen, für die einzelnen Prozesse verfügbaren und von ihnen benötigten Energie zurückgeführt werden. -

8. Zum Schluß sei noch darauf hingewiesen, daß früher besprochene Befunde zur Hutbildung eine entscheidende Wirkung des Cytoplasmas auf den Kern erkennen lassen, obwohl andererseits eine unmittelbare und mittelbare Kernabhängigkeit der vorbereitenden Hutbildungsprozesse aufgezeigt werden konnte ${ }^{1,4}$. Diese Schlüsse stehen in Einklang mit Versuchen, welche Beziehungen zwischen Stielwachstum und Kernentwicklung betreffen und besonders die Bedeutung des cytoplasmatischen Energiezustandes zeigen ${ }^{9}$. Ferner ergeben biochemische Untersuchungen die große Bedeutung des Cytoplasmas ${ }^{10}$. Schließlich ist zu erwähnen, daß auch für den dritten der hier betrachteten Vorgänge, die Auslösung der Kernteilung, eine cytoplasmatische Wirkung schon seit längerer Zeit bekannt ist. Werden bei A. mediterranea kleine Hüte auf Zellen mit teilungs-bereitem Kern transplantiert, so tritt die Kernteilung verzögert ein, umgekehrt erfolgt verfrühte Teilung, wenn ein kleiner Hut durch einen großen ersetzt wird. Die Kernteilung ist auch verzögert, wenn Zellen mit Hut durch rechtzeitige Amputation zu erneuter Stiel- und anschließend Hutbildung veranlaßt werden; sie erfolgt erst, wenn der neue Hut eine bestimmte Größe erreicht hat. Die Hutamputation kann mindestens 10-mal fortgesetzt werden. Dadurch kann - analog den Befunden an daueramputierten Amöben ${ }^{11}$ das System offenbar dauernd im Wachstum gehalten und die Kernteilung vollkommen unterdrückt werden (s. l. c. ${ }^{2}$ und eigene unveröffentl. Versuche).

Weitere Versuche werden aufklären müssen, welche stofflichen und energetischen Bedingungen im Cytoplasma charakteristisch und Voraussetzung für das Stielwachstum, die Hutbildung und die Kernteilung

9 J. H ä m m e r l in g, 8 me Congr. Intern. Botan. Paris 1954, im Druck; Exp. Cell Res. im Druck; H. S t i ch, Chromosoma, im Druck; G. W e r z, Planta, im Druck.

10 J. B r a c h e t, Colston Papers VII, 91, London 1954. 11 M. H a r tm a n n, Biol. Zbl. 42, 364 [1922]. 
sind. Hierbei wird man u. a. auch prüfen müssen, warum die Kernteilung normalerweise erst nach der Hutbildung einsetzt, obwohl sie offenbar einen geringeren Energiebedarf als letztere hat. Vielleicht kann in diesem speziellen Fall schon die Untersuchung der Kernentwicklung verschiedener Arten wie Acet. mediterranea und Acicularia bei bestimmten Umweltbedingungen etwas weiterführen.

\title{
Synthese und Abbau der Polyphosphate von Acetabularia nach autoradiographischen Untersuchungen des ${ }^{32}$ P-Stoffwechsels*
}

\author{
Von Hans Stich \\ Aus dem Max-Planck-Institut für Meeresbiologie, Abt. Hämmerling, Wilhelmshaven \\ (Z. Naturforschg. 10 b, 281-284 [1955]; eingegangen am 19. Januar 1955)
}

Mit Hilfe von ${ }^{32} \mathrm{P}$ wurde der Einbau und Ausbau von Phosphor in die im Cytoplasma liegenden Polyphosphatgrana von Acetabularia mediterranea bestimmt. Im Verhältnis zu der langen Lebensdauer der Zelle haben die Polyphosphate einen sehr schnellen Stoffumsatz. Wie aus Verdunkelungs- und Giftexperimenten (2.4-Dinitrophenol und Trypaflavin) hervorgeht, ist der Einbau von ${ }^{32} \mathrm{P}$ in die Polyphosphate vom normalen Ablauf der oxydativen Phosphorylierung abhängig.

Psisting olyphosphate (hochpolymerisierte Metaphosphate) scheinen bei phylogenetisch tief stehenden Organismen weit verbreitet zu sein: in Bakterien ${ }^{1,2,3}$, in Algen $^{4,5}$ sowie niederen ${ }^{6}$ und höheren ${ }^{7}$ Pilzen. Außer diesen Gruppen wurden sie nur, und auch da ausnahmsweise, bei Insekten nachgewiesen ${ }^{8}$. Die Fermente hingegen, die Polyphosphate in vitro $\mathrm{zu}$ spalten vermögen, finden sich sowohl in diesen als auch in Organismen, bei denen nach heutiger Ansicht keine Polyphosphate vorkommen. So konnte z. B. G ö t t e ${ }^{9}$ den Nachweis erbringen, daß in den Säugetierkörper gebrachte Polyphosphate durch Leber und Milz gespalten werden können.

Die Ausbildung und der Abbau der Polyphosphate enthaltenden Grana bei der einzelligen Grünalge Acetabularia ist vom Zustand der Zelle abhängig. Aus Verdunklung-, Regenerations- und TrypaflavinExperimenten wurde geschlossen, daß die Synthese im wesentlichen vom Ablauf der Photosynthese, der Abbau durch einen Verbrauch während des Zellstoffwechsels erfolgt ${ }^{5}$. Diese Versuche wurden fortgesetzt und erweitert, wobei der Umsatz der Polyphosphate mit Hilfe von ${ }^{32} \mathrm{P}$ autoradiographisch bestimmt

* Mit Unterstützung der Deuts chen Forschungsg e meins ch a f t.

1 J. Ebel, C. R. hebd. Séances Acad. Sci. 228, 1312 [1949].

2 G. Bringmann, Zbl. Bakteriol., Parasitenkunde, Infektionskrankh. Hyg., I. Abt., Orig. 156, 493 [1950].

$3 \mathrm{~F}$. Winder u. J. Denneny, Nature [London] 174, 353 [1954].

4 H.Albaum, A. Achatz, S.Hutner u. A. Hirschfeld, Arch. Biochemistry 29, 210 [1950].

5 H.Stich, Z. Naturforschg. 8 b, 36 [1953].

wurde. Eine Analyse der Wirkung von 2.4-Dinitrophenol und Verdunklung auf die Einlagerung von ${ }^{32} \mathrm{P}$ in die Polyphosphate sollte einen weiteren Einblick in die Abhängigkeit der Polyphosphatsynthese bringen, um so eine Vorstellung über die noch unklare Funktion dieses Stoffes im Zellhaushalt zu erlangen.

\section{Material und Methode}

Für die Versuche wurde Acetabularia mediterranea verwendet, die nach der von $\mathrm{Hämmerling} 10$ beschriebenen Methode kultiviert wird. Die Aktivierung der Zellen mit ${ }^{32} \mathrm{P}$ wurde schon früher genau beschrieben, so daß eine weitere Erörterung sich erübrigt 11. Für den autoradiographischen Nachweis des ${ }^{32} \mathrm{P}$ wurde die von Pelc12 ausgearbeitete Technik mit den „Kodak Autoradiographic Plates“ angewandt**. Die Polyphosphate der Grana des Cytoplasmas wurden nach den von $\mathrm{S} \mathrm{ti} \mathrm{ch}{ }^{5}$ aufgeführten Methoden nachgewiesen.

\section{Photosynthese und Ausbildung der Polyphosphatgrana}

In einer vorangegangenen Untersuchung konnte gezeigt werden, daß nach Verdunklung der Acetabu-

6 J. Wi ame, Biochim. biophysica Acta [Amsterdam] 1, 234 [1947].

7 T. Mann, Biochem. J. 38, 339 [1945].

8 St. Niemierko u. W. Niemierko, Nature [London] 166, 268 [1950].

9 H. Götte, Z. Naturforschg. 8 b, 173 [1953].

10 J. Hämmerling, Arch. Protistenkunde 97, 7 [1944].

$11 \mathrm{~J}$. Hämmerling u. H. Stich, Z. Naturforschg. 9 b, 199 [1954].

12 S. Pelc, Nature [London] 160, 749 [1949].

** Herr P e l c gab Herrn $\mathrm{H}$ ä m m e r li ng während eines Aufenthaltes in London dankenswerte praktische Informationen. 